\title{
Necessary and sufficient detector-efficiency conditions for the Greenberger-Horne-Zeilinger paradox
}

Jan-Åke Larsson

The self-archived postprint version of this journal article is available at Linköping University Institutional Repository (DiVA):

http:// urn.kb.se/ resolve?urn=urn:nbn:se:liu:diva-38612

N.B.: When citing this work, cite the original publication.

Larsson, J., (1998), Necessary and sufficient detector-efficiency conditions for the Greenberger-HorneZeilinger paradox, Physical Review A. Atomic, Molecular, and Optical Physics, 57(5), R3145-R3149. https:// doi.org/ 10.1103/PhysRevA.57.R3145

Original publication available at:

https:// doi.org/ 10.1103/PhysRevA.57.R3145

Copyright: American Physical Society

http:// www.aps.org// 


\title{
Physical Review A \\ ATOMIC, MOLECULAR, AND OPTICAL PHYSICS
}

\section{RAPID COMMUNICATIONS}

The Rapid Communications section is intended for the accelerated publication of important new results. Since manuscripts submitted to this section are given priority treatment both in the editorial office and in production, authors should explain in their submittal letter why the work justifies this special handling. A Rapid Communication should be no longer than 4 printed pages and must be accompanied by an abstract. Page proofs are sent to authors.

\section{Necessary and sufficient detector-efficiency conditions for the Greenberger-Horne-Zeilinger paradox}

\author{
Jan-Åke Larsson* \\ Department of Mathematics, Linköping University, S-581 83 Linköping, Sweden
}

(Received 9 December 1997)

\begin{abstract}
In this paper detector efficiency conditions are derived for the Greenberger-Horne-Zeilinger (GHZ) paradox. The conditions will be necessary and sufficient, i.e., the GHZ paradox is explicable in terms of a local-variable model if the efficiency is below the bounds, and the GHZ prerequisites are inconsistent at higher efficiencies. The derivation does not make use of any of the symmetry assumptions usually made in the literature, most notably the assumption of independent errors. The errors in local-hidden-variable models are governed by the "hidden variable" and, therefore, one cannot in general assume that the errors are independent. It will be shown that this assumption is not necessary. Moreover, bounds are presented that do not need the emission rate of particle triples to be known. An example of such a bound is the ratio of the triple coincidence rate and the double coincidence rate at two detectors, which needs to be higher than $75 \%$ to yield a contradiction. [S1050-2947(98)50305-8]
\end{abstract}

PACS number(s): 03.65.Bz

\section{INTRODUCTION}

The Greenberger-Horne-Zeilinger (GHZ) paradox [1-3] is often presented as the final argument against local variables, as it implies that any attempt to construct a localvariable model describing the GHZ setup inevitably results in a contradiction, i.e., the prerequisites (described below) are inconsistent. There is, however, one thing that obscures the issue somewhat-detector inefficiency. It is well known $[4,5]$ that the Bell inequality $[6]$ changes when detector inefficiency is present. The effect on the GHZ paradox has been studied [3], the bound on the detector efficiency being $90.8 \%$, but this bound would need the emission rate of particle triples to be known. In this paper this bound will be lowered somewhat, three bounds will be presented that do not need the emission rate to be known, and, moreover, the bounds presented will not depend upon independence of the errors. No other symmetry is assumed, such as constant efficiency or "fair sampling" $[3,7]$.

\footnotetext{
*Electronic address: jalar@mai.liu.se
}

The formalism used will follow Ref. [5] closely, and the spin terminology is chosen, although the path terminology in Ref. [3] may equally well be used. The sample space $\Lambda$ is the mathematical analogue to the state space used in physics, and a sample $\lambda$ is a point in that space corresponding to a certain value of the "hidden variable." The measurement results are described by random variables (RV's) $X(\lambda)$ that take their values in the value space $V$, instead of the quantum measurement operators. To be a probabilistic model a probability measure $P$ on the space $\Lambda$ is also needed.

Detector inefficiency is included in the model by stating that the RV $X$ might only be defined on a subset $\Lambda_{X}$ of the hidden-variable probability space $\Lambda$. The probability notation

$$
P_{X}(E)=P\left(E \mid \Lambda_{X}\right)
$$

will also be used to describe the conditional probability of the event $E$, given that the measurement $X$ has given a result. "Deterministic local variables" will be used, but the generalization to the "stochastic" case is straightforward.

There are in the GHZ setup three particles with three associated measurements, so there are three different RV's that 
are denoted as follows. The RV's describe measurement results from the first detector if it is unprimed $(A)$, from the second when primed $\left(B^{\prime}\right)$, and from the third when it has two primes $\left(C^{\prime \prime}\right)$. The detector orientations will be denoted $a, b$, and $c$.

The assumptions made on the model can be written as:

(i) Realism. Measurement results can be described by probability theory, using (three families of) RV's:

$$
\begin{aligned}
A(a, b, c): \Lambda_{A}(a, b, c) & \rightarrow V \\
\lambda & \mapsto A(a, b, c, \lambda) \\
B^{\prime}(a, b, c): \Lambda_{B^{\prime}}(a, b, c) & \rightarrow V \\
\lambda & \mapsto B^{\prime}(a, b, c, \lambda) \\
C^{\prime \prime}(a, b, c): \Lambda_{C^{\prime \prime}}(a, b, c) & \rightarrow V \\
\lambda & \mapsto C^{\prime \prime}(a, b, c, \lambda)
\end{aligned}
$$$$
\forall a, b, c \text {. }
$$

(ii) Locality. A measurement result should be independent of the detector orientation at the other particles:

$$
\begin{array}{cc}
A(a, \lambda) \stackrel{\operatorname{def}}{=} A(a, b, c, \lambda) & \text { on } \Lambda_{A}(a)=\Lambda_{A}(a, b, c) \\
& \text { independently of } b \text { and } c . \\
B^{\prime}(b, \lambda)=B^{\prime}(a, b, c, \lambda) & \text { on } \Lambda_{B^{\prime}}(b)=\Lambda_{B^{\prime}}(a, b, c) \\
& \text { independently of } a \text { and } c . \\
C^{\prime \prime}(c, \lambda)=C^{\prime \prime}(a, b, c, \lambda) & \text { on } \Lambda_{C^{\prime \prime}}(c)=\Lambda_{C^{\prime \prime}}(a, b, c) \\
& \text { independently of } a \text { and } b .
\end{array}
$$

(iii) Measurement result restriction. Only the results \pm 1 should be possible:

$$
V=\{-1,+1\}
$$

In the GHZ paradox only the $x$ and $y$ directions are used, and by the notation $X=A(x, \lambda), Y=A(y, \lambda), X^{\prime}=B^{\prime}(x, \lambda)$, and so on, the following will be shortened considerably. There are then only six different random variables to be used:

$$
X, X^{\prime}, X^{\prime \prime}, Y, Y^{\prime} \text {, and } Y^{\prime \prime} \text {, defined on }
$$

$\Lambda_{X}, \Lambda_{X^{\prime}}, \Lambda_{X^{\prime \prime}}, \Lambda_{Y}, \Lambda_{Y^{\prime}}$, and $\Lambda_{Y^{\prime \prime}}$, respectively.

The fourth and last assumption is a specification of the required measurement results.

(iv) GHZ requirement. The following measurement results should be obtained:

$$
\begin{array}{lll}
X Y^{\prime} Y^{\prime \prime}=1 & \text { on } & \Lambda_{X Y^{\prime} Y^{\prime \prime}}, \\
Y X^{\prime} Y^{\prime \prime}=1 & \text { on } & \Lambda_{Y X^{\prime} Y^{\prime \prime}}, \\
Y Y^{\prime} X^{\prime \prime}=1 & \text { on } & \Lambda_{Y Y^{\prime} X^{\prime \prime}},
\end{array}
$$

and

$$
X X^{\prime} X^{\prime \prime}=-1 \quad \text { on } \quad \Lambda_{X X^{\prime} X^{\prime \prime}}
$$

The set $\Lambda_{X Y^{\prime} Y^{\prime \prime}}$ equals quite naturally the set $\Lambda_{X} \cap \Lambda_{Y^{\prime}} \cap \Lambda_{Y^{\prime \prime}}$, the set where all three RV's are defined, and this shorthand notation will be used throughout the paper. These four prerequisites will be assumed to hold except on a set of zero probability (a null set).

It is easy to construct a three-particle quantummechanical state for which (iv) is satisfied,

$$
\frac{1}{\sqrt{2}}(|+++\rangle-|---\rangle)_{Z Z^{\prime} Z^{\prime \prime}}
$$

The inconsistency inherent in these four prerequisites is that if perfect detectors are assumed, then $\Lambda_{X}=\Lambda_{X^{\prime}}=\Lambda_{X^{\prime \prime}}=\Lambda_{Y}$ $=\Lambda_{Y^{\prime}}=\Lambda_{Y^{\prime \prime}}=\Lambda$, and by using in order from left to right, (iv), (iii), (ii), and (iv),

$$
\begin{aligned}
-1 & =X X^{\prime} X^{\prime \prime}=X X^{\prime} X^{\prime \prime} \cdot Y^{2} \cdot Y^{\prime 2} \cdot Y^{\prime \prime 2} \\
& =X Y^{\prime} Y^{\prime \prime} \cdot Y X^{\prime} Y^{\prime \prime} \cdot Y Y^{\prime} X^{\prime \prime}=1
\end{aligned}
$$

with probability 1 , so evidently assuming perfect detectors and (i)-(iv) except on a null set yields a contradiction.

\section{DETECTOR EFFICIENCY}

To be able to formulate the main result of the paper, a formal specification of the term efficiency is needed. There are many possible estimates of which four will be presented here for reasons that will become obvious. First, is the number that is instinctively associated with detector efficiency, the "single-particle efficiency"

$$
\eta_{1}=\min _{A, i} P\left(\Lambda_{A^{(i)}}\right) .
$$

The minimum is taken over all orientations of the detector $(A)$ and all detectors $(i)$, i.e., $X, X^{\prime}, X^{\prime \prime}, Y, Y^{\prime}$, and $Y^{\prime \prime}$. Note that neither $P\left(\Lambda_{X}\right)=P\left(\Lambda_{X^{\prime}}\right)$ nor any other symmetry is assumed, as it is desirable to use as few assumptions as possible; e.g., the "fair sampling" assumption [3,7] is not used. $\eta_{1}$ is then the lowest probability of the six possible sets.

A problem with the single-particle efficiency is that the triple emission rate is needed to estimate it. It would be desirable to have an estimate that is directly available in the raw coincidence data. Therefore, of all the remaining estimates, only the three that have this property will be presented. There is, however, a tradeoff as the simplification in extracting them from experiment makes them slightly more intricate to use formally. The "two-particle efficiency" is

$$
\eta_{2,1}=\min _{A, B, i \neq j} P_{A^{(i)}}\left(\Lambda_{B^{(j)}}\right),
$$

where again the minima are taken over all orientations of two of the detectors. Note that two different ones are used $(i \neq j)$; e.g., $P_{X}\left(\Lambda_{Y^{\prime \prime}}\right)$ is a valid combination but $P_{X}\left(\Lambda_{Y}\right)$ is not. The requirement of different particles will make it possible to extract information on $\eta_{2,1}$ from experiment by dividing the coincidence rate of two detectors in the experimental setup with the single detection rate of one of them. In this case there are 24 combinations (six possible $A^{(i)}$ 's and for each of these, four possible $B^{(j)}$ 's), and $\eta_{2,1}$ would be the 
lowest value obtained from these. A more formal note is that $\eta_{1}$ needs to be strictly positive for $\eta_{2,1}$ to be well-defined, but all these efficiency estimates will quite naturally be assumed to be strictly positive in the following. Finally there are two "three-particle efficiencies,",

$$
\eta_{3,2}=\min _{\substack{A, B, C, i \neq j \neq k \neq i}} P_{A^{(i)} B^{(j)}}\left(\Lambda_{C^{(k)}}\right)
$$

being one of them. Again the inequality should be valid for all possible orientations of the detectors corresponding to different particles; e.g., $P_{X Y^{\prime \prime}}\left(\Lambda_{X^{\prime}}\right)$ is a valid combination. This would be estimated by dividing the triple coincidence rate with the double coincidence rate at two detectors, and here there are also 24 combinations (six possible $A^{(i)}$ 's, four possible $B^{(j)}$ 's, and two possible $C^{(k)}$ 's give 48 combinations, but the ordering of $A^{(i)}$ and $B^{(j)}$ is unimportant; the result is 24 combinations). The other three-particle efficiency is

$$
\eta_{3,1}=\min _{\substack{A, B, C, i \neq j \neq k \neq i}} P_{A^{(i)}}\left(\Lambda_{\left.B^{(j)} C^{(k)}\right)}\right.
$$

which would correspond to dividing the triple coincidence rate with the detection rate at one detector (24 combinations).

\section{THE GHZ PARADOX WITH DETECTOR INEFFICIENCY}

To see what detector inefficiency would do to the GHZ paradox, a rather abstract theorem is necessary. Note that the proof is quite simple.

Theorem 1. Assuming (i)-(iv), except on a null set, yields a contradiction if and only if

$$
P\left(\Lambda_{X X^{\prime} X^{\prime \prime} Y Y^{\prime} Y^{\prime \prime}}\right)>0
$$

Proof. The proof is simply an observation in Eq. (4) that all of the RV's $X, X^{\prime}, X^{\prime \prime}, Y, Y^{\prime}$, and $Y^{\prime \prime}$ must be defined for the equality to be valid. But the set at which this is true is $\Lambda_{X X^{\prime} X^{\prime \prime} Y Y^{\prime} Y^{\prime \prime}}$.

If this is a null set, (iv) need not be satisfied on this set. Then Eq. (4) is no longer valid at any point of the probability space $\Lambda$ and there is no contradiction. Indeed, it is easy to construct a model satisfying (iv) on $\Lambda_{X X^{\prime} X^{\prime \prime} Y Y^{\prime} Y^{\prime \prime}}^{\mathrm{C}}$, the complement of $\Lambda_{X X^{\prime} X^{\prime \prime} Y Y^{\prime} Y^{\prime \prime}}$ (see, e.g., below). If the set has positive probability the contradiction remains.

This abstract theorem is mainly motivated by its use in the proof of the theorem below, but there is another motivation as well. In Ref. [5], the concept of change of ensemble was introduced, and was seen to be an important part of a localvariable model that was going to be used to approach the quantum-mechanical behavior in the Bell and ClauserHorne-Shimony-Holt (CHSH) inequalities. The above theorem states that the same concept is equally important in the GHZ setting, and more sharply so, because the statement is that, since the GHZ yields a full contradiction, the ensemble must change completely when changing the three detector orientations. The size of the "unchanged ensemble", $P_{X X^{\prime} X^{\prime \prime}}\left(\Lambda_{Y Y^{\prime} Y^{\prime \prime}}\right)$ must be zero, which is a substantial im- provement compared to the Bell and $\mathrm{CHSH}$ inequalities treated in Ref. [5], where it was allowed to be nonzero.

In the same way as in the Bell and CHSH settings, the change of ensemble is difficult to estimate from experiments, and a more direct comparison to experimental efficiency is necessary. Having specified the term "efficiency" above, we have the following theorem.

Theorem 2. Assuming (i)-(iv), except on a null set, yields a contradiction if at least one of the following is satisfied:

$$
\begin{gathered}
\eta_{1}>\frac{5}{6} \approx 83.33 \% \\
\eta_{2,1}>\frac{4}{5}=80 \% \\
\eta_{3,2}>\frac{3}{4}=75 \% \\
\eta_{3,1}>\frac{3}{5}=60 \%
\end{gathered}
$$

Furthermore, if none of (10a)-(10d) is satisfied, there exists a local-variable model satisfying (i)-(iv) that yields the quantum-mechanical statistics of the GHZ state in the |\rangle$_{X^{(i)}}$ and |\rangle$_{Y^{(i)}}$ bases except for detector inefficiency.

Proof. The proof begins with the first statement.

(a) Using $\eta_{1}$, a simple derivation yields

$$
\begin{aligned}
P\left(\Lambda_{X X^{\prime} X^{\prime \prime} Y Y^{\prime} Y^{\prime \prime}}\right)= & 1-P\left(\Lambda_{X}^{\mathrm{C}} \cup \Lambda_{X^{\prime}}^{\mathrm{C}} \cup \Lambda_{X^{\prime \prime}}^{\mathrm{C}} \cup \Lambda_{Y}^{\mathrm{C}} \cup \Lambda_{Y^{\prime}}^{\mathrm{C}} \cup \Lambda_{Y^{\prime \prime}}^{\mathrm{C}}\right) \\
\geqslant & 1-P\left(\Lambda_{X}^{\mathrm{C}}\right)-P\left(\Lambda_{X^{\prime}}^{\mathrm{C}}\right)-P\left(\Lambda_{X^{\prime \prime}}^{\mathrm{C}}\right)-P\left(\Lambda_{Y}^{\mathrm{C}}\right) \\
& -P\left(\Lambda_{Y^{\prime}}^{\mathrm{C}}\right)-P\left(\Lambda_{Y^{\prime \prime}}^{\mathrm{C}}\right) \\
= & P\left(\Lambda_{X}\right)+P\left(\Lambda_{X^{\prime}}\right)+P\left(\Lambda_{X^{\prime \prime}}\right)+P\left(\Lambda_{Y}\right) \\
& +P\left(\Lambda_{Y^{\prime}}\right)+P\left(\Lambda_{Y^{\prime \prime}}\right)-5 \\
\geqslant & 6 \eta_{1}-5 .
\end{aligned}
$$

(b) Using $\eta_{2,1}$ the same approach as above on $P_{X}\left(\Lambda_{X^{\prime} X^{\prime \prime} Y Y^{\prime} Y^{\prime \prime}}\right)$ is quite useless, since $\eta_{2,1}$ does not yield any estimate on probabilities where the same particle occurs twice, e.g., $P_{X}\left(\Lambda_{Y}\right)$. A slightly more sophisticated approach must be used to avoid probabilities with two occurrences of the same particle:

$$
\begin{aligned}
P_{X X^{\prime}}\left(\Lambda_{Y}\right) & =\frac{P_{X^{\prime}}\left(\Lambda_{X Y}\right)}{P_{X^{\prime}}\left(\Lambda_{X}\right)} \\
& =\frac{P_{X^{\prime}}\left(\Lambda_{X}\right)+P_{X^{\prime}}\left(\Lambda_{Y}\right)-P_{X^{\prime}}\left(\Lambda_{X} \cup \Lambda_{Y}\right)}{P_{X^{\prime}}\left(\Lambda_{X}\right)} \\
& \geqslant 1+\frac{\eta_{2,1}-1}{P_{X^{\prime}}\left(\Lambda_{X}\right)} \geqslant 1+\frac{\eta_{2,1}-1}{\eta_{2,1}}=2-\frac{1}{\eta_{2,1}} .
\end{aligned}
$$

If the set in the parentheses on the left-hand side is $\Lambda_{X^{\prime \prime}}$ or $\Lambda_{Y^{\prime \prime}}$, the same inequality holds. It also holds if the set is $\Lambda_{Y^{\prime}}$ (exchange $X$ with $X^{\prime}$ throughout the equation). Now the approach in Eq. (11) yields

$$
P_{X X^{\prime}}\left(\Lambda_{X^{\prime \prime} Y Y^{\prime} Y^{\prime \prime}}\right) \geqslant 4\left(2-\frac{1}{\eta_{2,1}}\right)-3=5-\frac{4}{\eta_{2,1}},
$$

and then, 


$$
\begin{aligned}
P\left(\Lambda_{X X^{\prime} X^{\prime \prime} Y Y^{\prime} Y^{\prime \prime}}\right) & =P_{X X^{\prime}}\left(\Lambda_{X^{\prime \prime} Y Y^{\prime} Y^{\prime \prime}}\right) P_{X}\left(\Lambda_{X^{\prime}}\right) P\left(\Lambda_{X}\right) \\
& \geqslant P_{X X^{\prime}}\left(\Lambda_{X^{\prime \prime} Y Y^{\prime} Y^{\prime \prime}}\right) \eta_{2,1} \eta_{1} \geqslant\left(5 \eta_{2,1}-4\right) \eta_{1} .
\end{aligned}
$$

(c) Using $\eta_{3,2}$ and the same approach as in (b),

$$
\begin{aligned}
& P\left(\Lambda_{X X^{\prime} X^{\prime \prime} Y Y^{\prime} Y^{\prime \prime}}\right) \\
& \quad=P_{X X^{\prime} X^{\prime \prime}}\left(\Lambda_{Y Y^{\prime} Y^{\prime \prime}}\right) P_{X X^{\prime}}\left(\Lambda_{X^{\prime \prime}}\right) P_{X}\left(\Lambda_{X^{\prime}}\right) P\left(\Lambda_{X}\right) \\
& \quad \geqslant P_{X X^{\prime} X^{\prime \prime}}\left(\Lambda_{Y Y^{\prime} Y^{\prime \prime}}\right) \eta_{3,2} \eta_{2,1} \eta_{1} \geqslant\left(4 \eta_{3,2}-3\right) \eta_{2,1} \eta_{1} .
\end{aligned}
$$

(d) Using $\eta_{3,1}$ the result is

$$
\begin{aligned}
P\left(\Lambda_{X X^{\prime} X^{\prime \prime} Y Y^{\prime} Y^{\prime \prime}}\right) & =P_{X X^{\prime} X^{\prime \prime}}\left(\Lambda_{Y Y^{\prime} Y^{\prime \prime}}\right) P_{X}\left(\Lambda_{X^{\prime} X^{\prime \prime}}\right) P\left(\Lambda_{X}\right) \\
& \geqslant P_{X X^{\prime} X^{\prime \prime}}\left(\Lambda_{Y Y^{\prime} Y^{\prime \prime}}\right) \eta_{3,1} \eta_{1} \geqslant\left(5 \eta_{3,1}-3\right) \frac{\eta_{1}}{2}
\end{aligned}
$$

From the inequalities (11), (14), (15), and (16) we have $P\left(\Lambda_{X X^{\prime} X^{\prime \prime} Y Y^{\prime} Y^{\prime \prime}}\right)>0$, if at least one of (10a)-(10d) is satisfied (and both $\eta_{2,1}$ and $\eta_{1}$ are strictly positive). Using Theorem 1, the first statement follows.

The second statement will be proved by construction; the model will of course be contradiction free. Let $\Lambda$ (the probability space) be a point set with 48 different points, and let the points have equal probability $\left(\frac{1}{48}\right)$. At the first 16 points the RV's are assigned signs (remember that $V=\{-1,+1\}$ ) from the following two tables, where $\nexists$ denotes that the corresponding $\mathrm{RV}$ is undefined at this point.

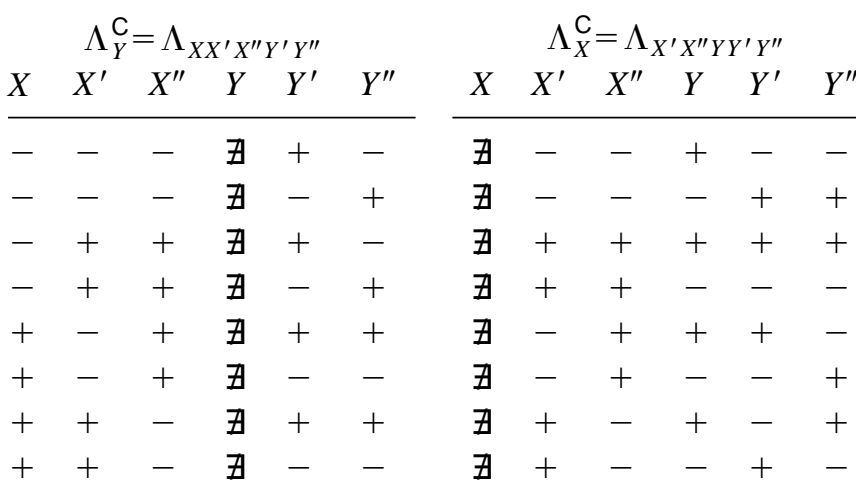

The values at the remaining 32 points are obtained by interchange of the first and second particles $\left(X \leftrightarrows X^{\prime}\right.$, $\left.Y \leftrightarrows Y^{\prime}\right)$ and by interchange of the first and third particles ( $X \leftrightarrows X^{\prime \prime}, \quad Y \leftrightarrows Y^{\prime \prime}$ ). Interchange of the second and third particles $\left(X^{\prime} \leftrightarrows X^{\prime \prime}, \quad Y^{\prime} \leftrightarrows Y^{\prime \prime}\right)$ corresponds to a reordering of the rows in the table. By construction the model is then symmetric with respect to interchange of the particles in the sense that the probabilities are the same. $\Lambda$ then consists of six disjunct sets of eight points each, at which one of the RV's is undefined.

This yields $P\left(\Lambda_{A^{(i)}}\right)=\frac{5}{6}$ for all RV's, i.e., $\eta_{1}=\frac{5}{6}$, and the others are obtained by rather simple checks: $\eta_{2,1}=\frac{4}{5}, \eta_{3,2}$ $=\frac{3}{4}$, and $\eta_{3,1}=\frac{3}{5}$; e.g.,

$$
\begin{gathered}
P\left(\Lambda_{Y Y^{\prime} Y^{\prime \prime}}\right)=P\left(\Lambda_{X}^{\mathrm{C}}\right)+P\left(\Lambda_{X^{\prime}}^{\mathrm{C}}\right)+P\left(\Lambda_{X^{\prime \prime}}^{\mathrm{C}}\right)=\frac{3}{6}, \\
P\left(\Lambda_{Y^{\prime} Y^{\prime \prime}}\right)=P\left(\Lambda_{Y Y^{\prime} Y^{\prime \prime}}\right)+P\left(\Lambda_{Y}^{\mathrm{C}}\right)=\frac{4}{6}
\end{gathered}
$$$$
\Rightarrow
$$

$$
P_{Y^{\prime} Y^{\prime \prime}}\left(\Lambda_{Y}\right)=\frac{3}{4}
$$

By inspection $X Y^{\prime} Y^{\prime \prime}=1=-X X^{\prime} X^{\prime \prime}$ in $\Lambda_{Y}^{\mathrm{C}}$, and $Y X^{\prime} Y^{\prime \prime}$ $=1=Y Y^{\prime} X^{\prime \prime}$ in $\Lambda_{X}^{\mathrm{C}}$, and by using the symmetry, (iv) follows. Indeed, all the statistics in the |\rangle$_{X^{(i)}}$ and |\rangle$_{Y^{(i)}}$ bases, obtained from the quantum-mechanical state in Eq. (3), are possible to derive more or less by inspection, except of course for detector inefficiency (note that this is not a complete model of the quantum-mechanical state as only the $X^{(i)}$ and $Y^{(i)}$ measurement results are specified). This completes the proof.

\section{CONCLUSIONS}

The result is that an experiment refuting local variables using the GHZ paradox does so if and only if the efficiency is higher than the bounds in Theorem 2 derived above:

$$
\begin{gathered}
\eta_{1}>\frac{5}{6} \approx 83.33 \% \\
\eta_{2,1}>\frac{4}{5}=80 \% \\
\eta_{3,2}>\frac{3}{4}=75 \% \\
\eta_{3,1}>\frac{3}{5}=60 \%
\end{gathered}
$$

An experiment at lower efficiencies would not be conclusive, as it is possible to construct a local-variable theory describing it.

As to the lowest bound, $\eta_{3,1}>60 \%$ seems to be lowest, but this estimate of the efficiency corresponds to adding two detectors to the measurement setup, so it is only natural that this bound would be low. One would naively expect $\eta_{3,1}$ $=\eta_{3,2} \eta_{2,1}$, but this need not be generally true given only the definitions of the efficiency estimates [(6)-(8)], since no additional symmetries are imposed. The bounds, however, do seem to follow this rule as $60 \%$ is $75 \%$ of $80 \%$, and the model given in the latter part of the proof of Theorem 2 does indeed possess the symmetries necessary for this to hold.

Another important note is that the bound derived does not need independent errors. The errors are decided by the local variable and could in principle be dependent on each other, but in Theorem 2 this is not a problem, as it is valid anyway. If one were to assume independent errors with the efficiency $\eta$, the result is that $\eta_{1}=\eta_{2,1}=\eta_{3,2}=\eta$, and $\eta_{3,1}=\eta^{2}$. The bound would then be $\eta=\eta_{3,2}>75 \%$.

Thus, the most important bound would be when adding the last detector to the measurement setup: $\eta_{3,2}>75 \%$. This bound is valid quite generally, since no symmetries are assumed in the proof. 
[1] D. M. Greenberger, M. A. Horne, and A. Zeilinger, in Bell's Theorem, Quantum Theory, and Conceptions of the Universe, edited by M. Kafatos (Kluwer Academic, Dordrecht, 1989).

[2] N. D. Mermin, Phys. Today 43 (6), 9 (1990).

[3] D. M. Greenberger, M. A. Horne, A. Shimony, and A. Zeilinger, Am. J. Phys. 58, 1131 (1990).
[4] A. Garg and N. D. Mermin, Phys. Rev. D 35, 3831 (1987).

[5] J.-A. Larsson, Phys. Rev. A 57, 3304 (1998).

[6] J. S. Bell, Physics (Long Island City, NY) 1, 195 (1964).

[7] J. F. Clauser, M. A. Horne, A. Shimony, and R. A. Holt, Phys. Rev. Lett. 23, 880 (1969). 